\title{
Fetomaternal Outcome of Pregnant Women Infected with Tuberculosis: An Analytical Study
}

\author{
Preeti F Lewis ${ }^{1}$, Amita S Budhewar ${ }^{2}$, Nitin Bhimrao Bavdekar ${ }^{3}$
}

\begin{abstract}
Background:Tuberculosis (TB) remains a major global public health problem, despite that maternal TB remains an obscure and underestimated issue. The prevalence of TB in pregnancy is not well known. The present study is aimed to determine the prevalence of TB and assess the obstetrical and perinatal outcomes of TB in antenatal women.

Materials and methods: A prospective observational study was done in a tertiary care center over a period of 1 year from January 2019 to January 2020. Pregnant women in the age-group of 18-42 years diagnosed with TB in the antenatal period were included in our study for evaluating maternal and fetal outcomes. We excluded those antenatal women with a history of TB and adequately treated and cured and those women with TB who did not deliver during the study period. Data collected from individual case record included age, parity, socioeconomic status, time of diagnosis, and complication in antenatal, intrapartum, and postpartum period. The mean birth weight of infants and frequency of small for gestation, stillbirth, neonatal depression, neonatal intensive care unit (NICU) admission, and neonatal death were noted, and perinatal outcome was seen.

Results: Five-thousand and two hundred patients delivered during the study period; among those, 70 diagnosed with TB in the antenatal period were included, making a prevalence rate of 1.34/1,000 per pregnant woman. There was a significant increase in the incidence of preterm delivery, anemia, and intrauterine growth restriction in TB. In our study, we found that maternal and perinatal outcomes in pregnant women with TB depend on site, the severity of disease, gestation at diagnosis, and complication of TB.

Conclusion: The prevalence of TB in antenatal women at our institute was 1.34/1,000 per pregnant woman. A high suspicion is required to acknowledge the varying disease spectrum and thus complications of TB in antenatal women and initiate treatment early for better outcomes. Keywords: Antenatal women, Maternal death, NICU admission, Perinatal outcome, Preterm birth, Stillbirth, Tuberculosis.

Journal of South Asian Federation of Obstetrics and Gynaecology (2021): 10.5005/jp-journals-10006-1948
\end{abstract}

\section{INTRODUCTION}

Tuberculosis (TB) remains a major global public health problem, despite that maternal TB remains an obscure and underestimated issue. It is estimated that one-fourth of the global population is exposed to TB, of which $5-10 \%$ develop active disease in their lifetime. ${ }^{1}$ Worldwide 10 million people were infected with TB in 2018. The burden of this disease varies enormously among countries from fewer than $5-500$ new cases per $10,000 .^{2}$

The real prevalence of TB in the Indian community is yet not known precisely and is probably underestimated. This can be due to nonspecific clinical features, poor diagnostic tools for confirmation of the disease, absence of a standardized system for notification of the disease, large population exposure for the disease, population under poverty line with malnutrition and high vulnerability to $\mathrm{TB}$, lack of understanding in the community for seeking early and complete treatment, associated social disgrace with the infection, and high prevalence of coinfection with human immunodeficiency virus (HIV). ${ }^{3}$ India contributes to nearly $21 \%$ of the global burden of TB among pregnant women, and therefore, the estimated prevalence of TB stands at 2.3 per 1,000 pregnant women, translating to about 44,500 patients annually. ${ }^{4,5}$ Numerous studies show there is a significant increase in the risk of active TB infection seen in both pregnant and postnatal period in high burden countries like India although many reports are conflicting too. ${ }^{6}$

Pulmonary TB is the commonest site for contamination; anyway these days extrapulmonary TB is getting more pervasive. It has been seen that there is a late in the diagnosis of TB in pregnancy due to various symptoms with which patients present many times
${ }^{1}$ Department of Obstetrics and Gynaecology, Grant Government Medical College and JJ Group of Hospitals, Mumbai, Maharashtra, India

${ }^{2}$ Department of Obstetrics and Gynaecology, Grant Government Medical College, Mumbai, Maharashtra, India

${ }^{3}$ Department of Community Medicine, DPH, PGDHAM, PGDMLS MMHS, Maharshtra, India

Corresponding Author: Amita S Budhewar, Department of Obstetrics and Gynaecology, Grant Government Medical College, Mumbai, Maharashtra, India, Phone: +91 7447309363, e-mail: amitabudhewar25@gmail.com

How to cite this article: Lewis PF, Budhewar AS, Bavdekar NB. Fetomaternal Outcome of Pregnant Women Infected with Tuberculosis: An Analytical Study. J South Asian Feder Obst Gynae 2021;13(4):197-201.

Source of support: Nil

Conflict of interest: None

converging to physiological changes in pregnancy. ${ }^{7}$ TB is neither more frequent nor more serious in pregnancy than nonpregnant women. Women with TB increase the risk of neonatal death by six times and double the risk of theirs being born prematurely or with low birth weight. ${ }^{8}$

Several studies show that TB in pregnancy has been related to poor obstetrical, fetal, and perinatal outcome. But the early diagnosis of TB still remains the biggest obstacle in the management of the disease. ${ }^{9}$ In recent, postmortem analysis of maternal deaths 
brings out that infection, including TB, is an important contributor to maternal death in India. ${ }^{10}$

The present study is aimed to determine the prevalence of the TB and to evaluate the risks of adverse pregnancy outcomes in maternal TB.

\section{Materials and Methods}

This was a prospective observational study done in a tertiary care center over a period of 1 year from January 2019 to January 2020. The Institutional Ethics Committee approval was obtained. Antenatal women in the age-group of 18-42 years detected to have TB in the antenatal period were included in our study for evaluating maternal and perinatal outcomes. They were assessed for manifestation, including investigation such as sputum for acidfast bacillus and chest X-ray, particularly for those with clinically progressed pneumonic illness, subsequent to advising and taking consent that the radiation dosage $<0.01 \mathrm{mGy}$ is protected in pregnancy, gene expert, and further multidisciplinary approach involving microbiological and pulmonary medicine expert opinion. We excluded those antenatal women with history of TB and satisfactorily treated and cured and those women with TB who did not deliver during the study period. Data collected from individual case records included age, gravida score, time of diagnosis, and complication during the antenatal and postnatal period. The mean birth weights of infants and frequency of small for gestation, neonatal depression, neonatal death, Apgar score, NICU admission, and stillbirth were noted, and perinatal outcome was seen.

\section{Data Analysis}

The data were collected, compiled, and analyzed using EPI info (version 7.2). The qualitative variables were expressed in terms of percentages. The quantitative variables were both categorized and expressed in terms of percentages or mean and standard deviations. The difference between the two proportions was analyzed using Chi-square or Fisher's exact test. Student's $t$-test was used to test the difference between the two means.

\section{Compliance with Ethical Standards}

The research is in accordance with the ethical guidelines of the Institute's Ethical Committee. All observations done in this report are on humans and in accordance with the ethical guidelines of the Institute's Research Committee and compliance with the 1964 Helsinki declaration and its later amendments and ethical standard. Informed consent was taken from the participants involved in this study in their language.

\section{Results}

\section{Prevalence Rate}

In our study, 5,200 patients delivered during the study period; among those, 70 diagnosed with TB in the antenatal period were included, making prevalence rate of 13.4 per 1,000 pregnant women in our study; $52 / 70$ (74.28\%) were diagnosed with pulmonary TB, and $18 / 70(25.17 \%)$ cases were diagnosed with extrapulmonary TB.

\section{Age}

In our study, 34 (48.6\%) cases belong to an age-group of 21-25 years with the mean age of $21.57 \pm 4.22$ years.

\section{Socioeconomic Status}

In our study, $28 / 70(40 \%)$ cases belong to lower socioeconomic status, and $42 / 70(60 \%)$ antenatal women belong to middle socioeconomic status.

\section{Registrations Status}

In our study, 46/70 (66\%) were registered and 24/70 (34\%) were unregistered antenatal women.

\section{Distribution as per Site of TB}

Figure 1 depicts the site of distribution of TB. Out of total 70 cases in the present study, 52 (74.28\%) women had pulmonary TB and $18(25.71 \%)$ were diagnosed with extrapulmonary TB, inclusive of tubercular lymphadenitis (13\%), abdominal TB (6\%), TB meningitis (4.16\%), intestinal TB (1.42\%), and Pott's spine (1.42\%).

\section{Antenatal Clinical Presentation}

Clinical profile of antenatal women was shown in Table 1. Chronic cough with expectoration was the most common presenting symptom in $49 / 70$ (70\%) followed by fever in $41 / 70$ (58.57\%); other symptoms like breathlessness, fatigue, and hemoptysis in $24 / 70$ (34.29\%); and weight loss in 10/70 (14.49\%), whereas some presented with atypical symptoms like headache, vomiting, and neck swelling.

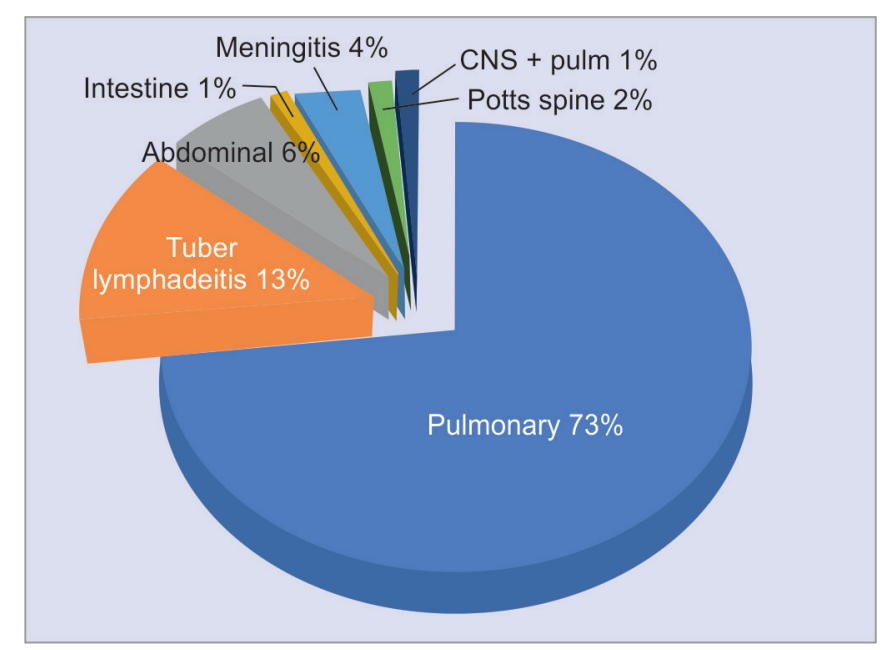

Fig. 1: Distribution as per the site of TB

Table 1: Clinical profile of subjects

\begin{tabular}{lcc}
\hline Symptoms & Seen in patients & Percentage \\
\hline Chronic cough with expectoration & 49 & 70.00 \\
Fever & 41 & 58.57 \\
Other symptoms (breathlessness, & 24 & 34.29 \\
fatigue hemoptysis, and loss of & & \\
appetite) & 10 & 14.29 \\
Weight loss & 4 & 5.71 \\
Headache, vomiting, and & & \\
convulsions & 3 & 4.29 \\
Neck swelling & 3 & 4.29 \\
Pain in abdomen and diarrhea & & \\
\hline
\end{tabular}




\section{Obstetric Complication}

Figure 1 describes the complications associated with TB. Among obstetric complications, preterm labor (18.51\%) followed by anemia (15.71\%) and intrauterine growth restriction (IUGR) (10\%) was the most common complications associated with TB in pregnancy.

\section{Pregnancy Outcome}

Figure 2 depicts the knowledge about the pregnancy outcome. Fourteen (20.28\%) females underwent spontaneous preterm vaginal delivery. Forty-two (60.86\%) had full-term normal delivery, and $10(14.49 \%)$ underwent cesarean section with the most common indication being fetal distress. Three had a spontaneous abortion. One antenatal woman succumbed to acute respiratory distress syndrome due to pulmonary TB at 5 months of gestation.

\section{Baby Weight Groups}

Thirty-five out of 66 (63.02\%) neonates had low birth weight, that is, less than $2.5 \mathrm{~kg} ; 11$ (16.66\%) had very low birth weight, that is, less than $1.5 \mathrm{~kg}$; and twenty had normal birth weight, that is, more than $2.5 \mathrm{~kg}$.

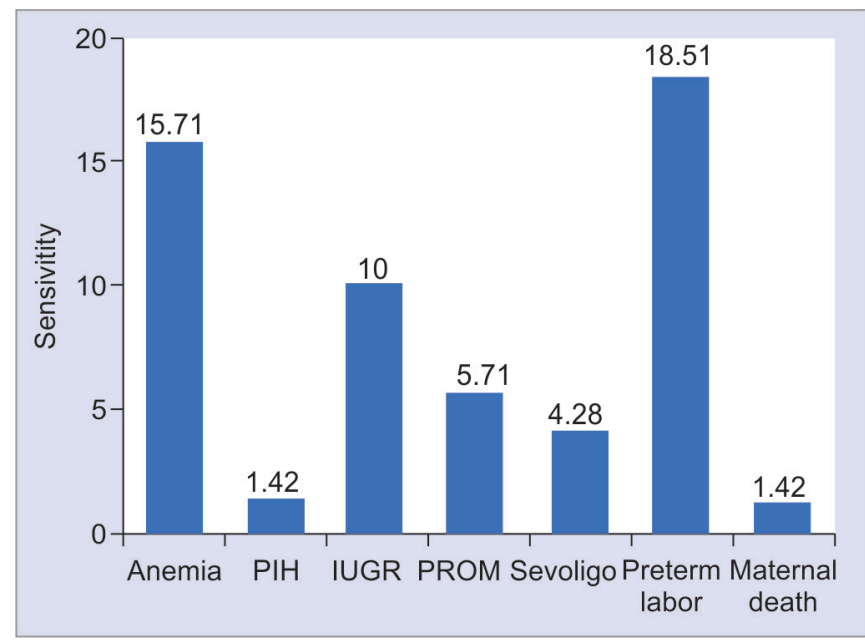

Fig. 2: Obstetric complications in women with TB in pregnancy

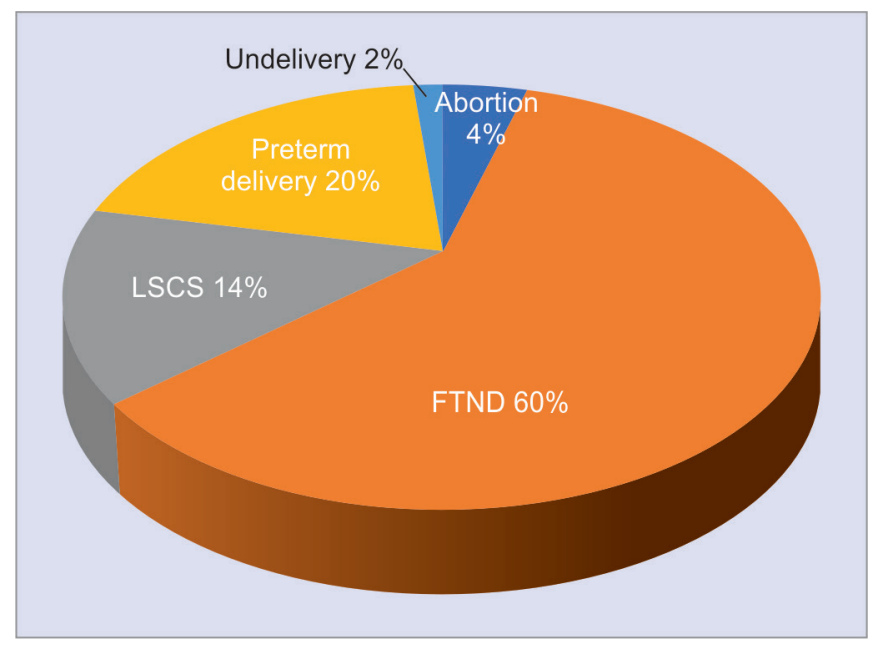

Fig. 3: Pregnancy outcome of tuberculosis

\section{NICU Admission of Babies of TB Patients}

Figures 3 and 4 describe the neonatal outcome in terms of NICU admission. The most common reason for NICU admission was prematurity (38\%) followed by low birth weight (31\%).

\section{Maternal Mortality}

There were eight (11.42\%) maternal mortalities observed in our study, of which five $(9.61 \%)$ were due to pulmonary TB and its complication and three (16.66\%) were due to extrapulmonary TB and its complications.

\section{Correlation of Pregnancy Outcome in Type of TB}

Significant correlation of type of TB and pregnancy outcome was shown in Table 2. There was a significant association between neonatal deaths and extrapulmonary TB $(p=0.031)$. Neonatal deaths were seen more in extrapulmonary TB group with seven neonatal deaths (38.89\%) compared to six (11.76\%) neonatal mortalities in the pulmonary TB group.

\section{Discussion}

TB is a globally disregarded medical problem, particularly in India. The World Health Organization assessed that India accounts for about $21 \%$ of the global burden in $2018 .^{2}$ The prevalence of TB in pregnancy ranges from 0.06 to $0.25 \%$ in low burden areas, whereas in high burden areas, it is $0.07-0.5 \%$ in HIV-negative pregnant women. This exceeds as high as $0.7-11 \%$ in HIV-positive females. ${ }^{11}$ In our study, the prevalence rate of TB is $1.34 \%$.

In our study, 52/70 (74.28\%) were diagnosed with pulmonary TB and 18/70 (25.17\%) were diagnosed with extrapulmonary TB as compared to $62 \%$ of extrapulmonary TB cases in a study conducted by Chopra et al., ${ }^{12} 66.6 \%$ by Yadav et al. ${ }^{7}$ and team, and $20 \%$ as reported by Jana et al. ${ }^{9}$

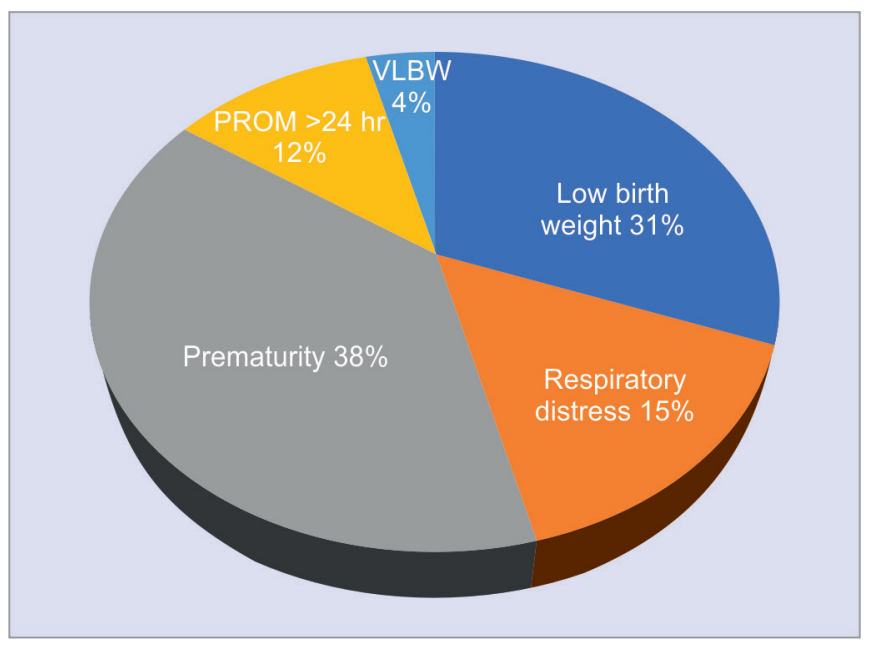

Fig. 4: Neonatal outcome according to NICU admission

Table 2: Correlation of pregnancy outcome with respect to the type of TB

\begin{tabular}{lcccc}
\hline & \multicolumn{3}{c}{ Pregnancy outcome } & \\
\cline { 2 - 4 } Cases & Abortion & Live & NND & Total \\
\hline Extrapulmonary & 1 & 10 & 7 & 18 \\
Pulmonary & 2 & 43 & 6 & 51 \\
Total & 3 & 54 & 13 & 69 \\
\hline
\end{tabular}

$X^{2}=6.941, p=0.031$ significant 
In our study, the average age index was $21.57 \pm 4.22$ years, which is comparable to $25.7 \pm 4.22$ years and $28.7 \pm 3.9$ years in a study conducted by Chopra et al. ${ }^{12}$ and Yadav et al., respectively. ${ }^{7}$

In our study, 42 women were from lower socioeconomic status and 28 from middle socioeconomic status. According to Muniyandi's study, the prevalence of TB was higher in those who live below the poverty line. ${ }^{13}$ Similar result was seen in our study.

Clinical findings of active TB in antenatal women can be troublesome, and there is frequently a difficulty in diagnosis because of vague nonspecific clinical features related to the physiological response to pregnancy. ${ }^{14}$ In our study, chronic cough with expectoration was the most common presenting symptom in $49 / 70$ (70\%) followed by fever in $41 / 70$ (58.57\%) and weight loss in $10 / 70$ (14.49\%), whereas some presented with atypical symptoms like headache, vomiting, and neck swelling. Gould and Aronoff ${ }^{15}$ and associate and Newton ${ }^{16}$ found cough as a common symptom.

In extrapulmonary TB, sites were lymph node (13\%), abdominal TB (6\%), tubercular meningitis (4.16\%), Pott's spine (1.42\%), and intestinal TB (1.42\%). Similar distribution was seen in Gaifer study ${ }^{17}$ and Chopra et al. study, ${ }^{12}$ while Yadav et al. ${ }^{7}$ study found genital TB as the most common extrapulmonary site.

The effects of TB depend on various factors such as the severity of disease, site of TB, time of gestation at the time of diagnosis, HIV coinfection, treatment, and compliance to treatment. ${ }^{18}$ In our study, a higher incidence of antenatal complications like preterm labor, anemia, IUGR, and oligohydraminos has been reported. Early treatment of TB (beginning in first and second trimester) led to the better maternal outcome as compared to late diagnosis in the third trimester. ${ }^{19}$

According to Mahendru et al., ${ }^{20}$ Loto and Awowole, ${ }^{21}$ and Sengupta et al. studies, ${ }^{22}$ extrapulmonary associated with low birth weight, poor Apgar score, higher incidence of prematurity, intrauterine growth restriction, small for gestation, and increase in neonatal death rate were similar to the finding seen in our study.

In the present study, cesarean section rate was $14.28 \%$. According to BCOG meta-analysis, cesarean section rate was two times in women with TB, which is was not seen in our study. This may be due to differences in the study population. As active TB infection poses a great risk to antenatal women and their fetuses, TB in pregnancy must be diagnosed and treated with a full course of anti-tubercular therapy.

Women with TB can breastfeed normally while taking antitubercular therapy. Current drug therapy is so efficacious that separation of the mother and infant is not recommended. ${ }^{23}$ Perinatal morbidity was similar in pregnant women receiving anti-tuberculous drugs from early pregnancy to that of uninfected women. We conclude that TB represents a risk factor for poor perinatal outcomes. TB with pregnancy is an important risk factor for adverse neonatal outcome. ${ }^{24}$ In our study, we found an increased risk of NICU admission, low birth weight, and perinatal mortality in pregnant women with TB.

In our study, eight maternal mortalities were reported; five maternal mortality due to pulmonary TB and its complication and three maternal mortality due to extrapulmonary TB and its complication. In our study, one woman was diagnosed with pulmonary TB at 3-month gestational age and started on antitubercular therapy. At 5 months of gestation, the patient presented with headache and convulsion and was diagnosed with tubercular meningitis after cerebrospinal fluid studies and computed tomography of the brain. The patient was admitted to ICU, but her condition deteriorated and could not revive.

In the present study, of 20 pregnant women diagnosed in the first trimester, only one had a stillbirth; while 14 antenatal women diagnosed with TB in the second trimester, one maternal mortality and 3 neonatal deaths were reported. In the third trimester, 14 antenatal women were diagnosed with TB, and of these, two stillbirth, one neonatal death, and five maternal mortality were reported. Early treatment of the disease in pregnancy regress its negative impact on perinatal outcome. ${ }^{25}$

\section{Conclusion and Recommendations}

The prevalence rate of TB in antenatal women at our institute during the study period is 13.4 per 1,000 pregnant women. Our study shows the higher risk of preterm labor, anemia, and intrauterine growth restriction with TB in pregnancy. We found that maternal and perinatal outcomes in pregnant women with TB depend on site, the severity of the disease, gestational age at diagnosis, complications of TB, and the onset of treatment. So, contraception should be eminently recommended to females in the reproductive age bracket with TB on treatment. Undiagnosed maternal TB remains a curse.

Early diagnosis of maternal TB is the biggest barrier in the management of TB in the antenatal period. So, clinicians, especially in high burden areas with limited resources like India, should employ available health assets like antenatal care programs to have patient-centered approach in diagnosis, counseling, and management of TB in the antenatal period. Perinatal TB is very hard to diagnose and can be life-threatening. All neonates born to TB mothers should be screened for TB, and placenta should be best studied for proof of TB. A high suspicion is required to acknowledge the varying disease spectrum and thus complications of TB, especially extrapulmonary TB in antenatal women, and initiate treatment early in pregnancy for better outcomes.

\section{References}

1. World Health Organization. WHO consolidated guidelines on tuberculosis: module 1: prevention: tuberculosis preventive treatment. In: WHO consolidated guidelines on tuberculosis: module 1: prevention: tuberculosis preventive treatment. World Health Organization; 2020.

2. World Health Organization. Global tuberculosis report 2019. Geneva (Switzerland): World Health Organization; 2019.

3. Baxi A, Neema H, Kadi P, et al. Prevalence of male genital tuberculosis in Indian infertile couples and its correlation with female genital tuberculosis. J South Asian Feder Obst Gynae 2016;8(1):13-15. DOI: 10.5005/jp-journals-10006-1377.

4. Vijayageetha M, Kumar AM, Ramakrishnan J, et al. Tuberculosis screening among pregnant women attending a tertiary care hospital in Puducherry, South India: is it worth the effort? Global Health Action 2019;12(1):1564488. DOI: 10.1080/16549716.2018.1564488.

5. Sugarman J, Colvin C, Moran AC, et al. Tuberculosis in pregnancy: an estimate of the global burden of disease. Lancet Glob Health 2014;2(12):e710-e716. DOI: 10.1016/S2214-109X(14)70330-4.

6. Jonsson J, Kühlmann-Berenzon S, Berggren I, et al. Increased risk of active tuberculosis during pregnancy and postpartum: a registerbased cohort study in Sweden. Eur Respir J 2020;55(3):1901886. DOI: 10.1183/13993003.01886-2019.

7. Yadav V, Sharma JB, Kachhawa G, et al. Obstetrical and perinatal outcome in pregnant women with extrapulmonary tuberculosis. Indian J Tuberc 2019;66(1):158-162. DOI: 10.1016/j.ijtb.2018.10.010. 
8. Banu EA, Nechita A, Elkan-Cojocaru EM, et al. Risk of tuberculosis in low birth weight children from East Romania. Arch Med Sci 2020;16(1):162. DOI: 10.5114/aoms.2018.78768.

9. Jana N, Vasishta K, Saha SC, et al. Obstetrical outcomes among women with extrapulmonary tuberculosis. New Engl J Med 1999;341(9): 645-649. DOI: 10.1056/NEJM199908263410903.

10. Panchabhai TS, Patil PD, Shah DR, et al. An autopsy study of maternal mortality: a tertiary healthcare perspective. J Postgrad Med 2009;55(1):8. DOI: 10.4103/0022-3859.48434.

11. Bates $M$, Ahmed $Y$, Kapata $N$, et al. Perspectives on tuberculosis in pregnancy. Int J Infect Dis 2015;32:124-127. DOI: 10.1016/ j.ijid.2014.12.014.

12. Chopra S, Siwatch S, Aggarwal N, et al. Pregnancy outcomes in women with tuberculosis: a 10-year experience from an Indian tertiary care hospital.TropDoct 2017;47(2):104-109.DOI: 10.1177/0049475516665765.

13. Muniyandi M, Ramachandran R. Socioeconomic inequalities of tuberculosis in India. Expert Opin Pharmacother 2008;9(10): 1623-1628. DOI: 10.1517/14656566.9.10.1623.

14. Sobhy S, Babiker ZOE, Zamora J, et al. Maternal and perinatal mortality and morbidity associated with tuberculosis during pregnancy and the postpartum period: a systematic review and meta-analysis. BJOG 2017;124(5):727-733. DOI: 10.1111/1471-0528.14408.

15. Gould JM, Aronoff SC. Tuberculosis and pregnancy-maternal, fetal, and neonatal considerations. Microbiol Spectr 2016;4(6):10.1128/ microbiolspec.TNMI7-0016-2016. DOI: 10.1128/microbiolspec.TNMI70016-2016.

16. Newton ER. Tuberculosis and pregnancy. Glob Libr Women's Med (ISSN: 1756-2228) 2008. DOI: 10.3843/GLOWM.10186.
17. Gaifer Z. Epidemiology of extrapulmonary and disseminated tuberculosis in a tertiary care center in Oman. Int J Mycobacteriol 2017;6(2):162. DOI: 10.4103/ijmy.ijmy_31_17.

18. Jana N, Barik S, Arora N, et al. Tuberculosis in pregnancy: the challenges for South Asian countries. J Obstet Gynaecol Res 2012;38(9):1125-1136. DOI: 10.1111/j.1447-0756.2012.01856.x.

19. Nguyen HT, Pandolfini C, Chiodini P, et al. Tuberculosis care for pregnant women: a systematic review. BMC Infect Dis 2014;14(1):617. DOI: 10.1186/s12879-014-0617-x.

20. Mahendru A, Gajjar K, Eddy J. Diagnosis and management of tuberculosis in pregnancy. Obstet Gynaecol 2010;12(3):163-171. DOI: 10.1576/toag.12.3.163.27598.

21. Loto OM, Awowole I. Tuberculosis in pregnancy: a review. J Pregnancy 2012;2012. DOI: 10.1155/2012/379271.

22. Sengupta $M$, Dasgupta $A$, Santra $D$, et al. Clinical manifestations and challenges in management of tuberculosis in pregnancy in a rural setting in Eastern India. J South Asian Feder Obst Gynae 2018;10(1):15-22. DOI: 10.5005/jp-journals-10006-1551.

23. World Health Organization, Stop TB Initiative (World Health Organization). Treatment of tuberculosis: guidelines. World Health Organization; 2010.

24. LaCourse SM, Greene SA, Dawson-Hahn EE, et al. Risk of adverse infant outcomes associated with maternal tuberculosis in a low burden setting: a population-based retrospective cohort study. Infect Dis Obstet Gynecol 2016;2016:6413713. DOI: 10.1155/2016/6413713.

25. Figueroa-Damian R, Arredondo-Garcia JL. Pregnancy and tuberculosis: influence of treatment on perinatal outcome. Am J Perinatol 1998;15(5):303-306. DOI: 10.1055/s-2007-993948. 